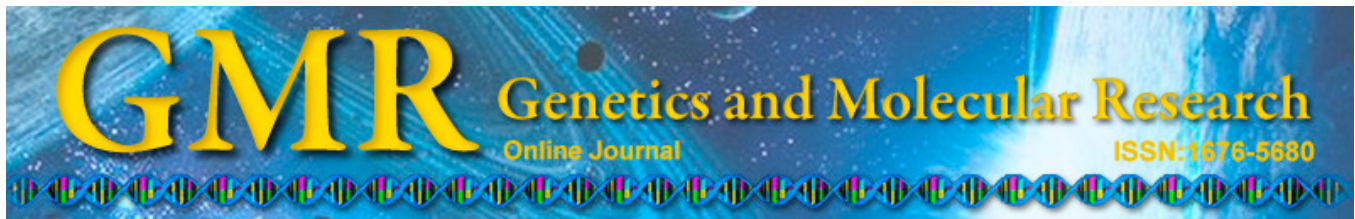

\title{
A novel 3-base pair deletion of the $C R Y A A$ gene identified in a large Chinese pedigree featuring autosomal dominant congenital perinuclear cataract
}

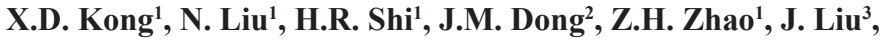 \\ J. Li-Ling ${ }^{3,4}$ and Y.X. Yang ${ }^{5}$ \\ ${ }^{1}$ Center of Prenatal Diagnosis, \\ The First Affiliated Hospital of Zhengzhou University, Zhengzhou, China \\ ${ }^{2}$ Department of Ophthalmology, \\ The First Affiliated Hospital of Zhengzhou University, Zhengzhou, China \\ ${ }^{3}$ Sino-Dutch Biomedical and Information School, \\ Northeastern University, Shenyang, China \\ ${ }^{4}$ Laboratory of Disease Genomics and Bioinformatics, \\ State Key Laboratory for Biotherapy, Sichuan University, Chengdu, China \\ ${ }^{5}$ Department of Emergency Medicine, \\ The First Affiliated Hospital of Zhengzhou University, Zhengzhou, China \\ Corresponding authors: X.D. Kong / Y.X. Yang \\ E-mail: kongxd@263.net/nc12000@126.com
}

Genet. Mol. Res. 14 (1): 426-432 (2015)

Received January 31, 2014

Accepted September 15, 2014

Published January 23, 2015

DOI http://dx.doi.org/10.4238/2015.January.23.16

\begin{abstract}
Congenital cataract is caused by reduced transparency of the lens resulting from metabolic disorders during the fetal period. The disease shows great heterogeneity both clinically and genetically. We identified a 4-generation ethnic Han Chinese family affected by autosomal dominant congenital perinuclear cataract. The patients underwent full clinical and ophthalmologic examinations to rule out any concomitant disorders. Blood samples were collected and genomic
\end{abstract}


DNA was extracted. Potential mutations in the candidate gene alpha A crystallin (CRYAA) were screened. Prenatal diagnosis was then provided for a fetus of the affected proband by chorionic villus sampling. In all patients, DNA sequencing of the CRYAA gene revealed a novel 3-bp deletion mutation in exon 3 (c.246_248delCGC), which led to deletion of codon 117 encoding arginine (p.117delR) in the peptide chain. The same mutation was not found among unaffected and healthy individuals. Bioinformatic analysis revealed that although the c.246_248delCGC is an 'in-frame' mutation, removal of arginine resulted in a significant change in the protein structure. The fetus did not possess this mutation and was confirmed to be healthy at 1-year follow-up. A novel diseasecausing mutation, c.246_248delCGC (p.117delR), of the CRYAA gene has been identified in a Chinese family with autosomal-type perinuclear congenital cataracts. This is also the first report of prenatal diagnosis of this type of congenital cataract.

Key words: Congenital perinuclear cataract; $C R Y A A$ gene; Gene mutation; Prenatal diagnosis

\section{INTRODUCTION}

Congenital cataract is caused by reduced transparency of the lens resulting from metabolic disorders during the fetal period. It is the second most common cause of blindness and amblyopia. The incidence of non-syndromic congenital cataract has been estimated to be 1-6 of every 10,000 newborns (Santana and Waiswo, 2011), for which genetic types account for approximately one-third (Lambert and Drack, 1996). Genetically, the disease features great heterogeneity, with at least 42 loci mapped to date (Hejtmancik and Smaoui, 2003). Various patterns in inheritance, including autosomal dominant, autosomal recessive, and X-linked, have been described, among which autosomal dominant is the most common. At least 14 genes have been associated with autosomal dominant congenital cataract (ADCC), including CRYAA, CRYAB, CRYBA1/A3, CRYBB1, CRYBB2, CRYGC, CRYGD, GJA8, GJA3, MIP, BFSP2, PITX3, HSF4, and MAF.

\section{MATERIAL AND METHODS}

\section{Patients}

We identified a large ethnic Han family from Henan, China, which included 10 patients from 4 generations ( 6 males and 4 females), affected with ADCC (Figure 1). The proband, aged 33 years, complained of blurred vision and poor eyesight since birth, but had no other eye discomfort. She received lens implantation, but gave birth to an affected girl in her first marriage. As she was pregnant again during her second marriage, she requested genetic counseling and prenatal diagnosis. Examination of her mother, who did not receive lens implantation, revealed central opacities, mild fetal nucleus opacity, and peripheral annular zones of opacities. The patient was without other ocular and systemic disease. All other affected individuals from the family have similar bilateral perinuclear cataracts but no systemic disease. 


\section{DNA extraction}

After obtaining informed consent, peripheral venous blood samples were taken from available family members. DNA was extracted using a DP318 kit (Tiangen Biotech, Beijing, China). For prenatal diagnosis, a chorionic villus sample was taken at the 11th gestational week. Fetal DNA was extracted with a DP316 kit (Tiangen Biotech).

\section{Polymerase chain reaction (PCR) and DNA sequencing}

Potential mutations in the alpha A crystallin gene CRYAA were sequenced by PCR and Sanger sequencing using primers described elsewhere (Guo et al., 2011). The lengths of the amplicons $(584,550$, and $551 \mathrm{bp})$ were verified on a $1.5 \%$ agarose gel, and then purified and sequenced bi-directionally using an ABI3130XL sequencer (Applied Biosystems, Foster City, CA, USA). The readings were then aligned with released sequences from the National Center for Biotechnology Information's GenBank database.

\section{Bioinformatic analysis}

The structures of the wild-type and mutant proteins were predicted using SWISSMODEL (http://beta.swissmodel.expasy.org/) (Arnold et al., 2006).

\section{Nomenclature of mutations and verification}

Sequence variants were initially searched against PubMed (http://www.ncbi.nlm.nih. gov/PubMed) and the MMA database (http://www.genomed.org/lovd). All new mutations were verified against corresponding sequences from 200 healthy unrelated individuals to rule out polymorphisms. New mutations were named according to the "Recommendations for the Description of Sequence Variants" guidelines published by the Human Genome Variation Society (http://www.hgvs.org/mutnomen/recs.html).

\section{Prenatal diagnosis}

Following the detection and verification of the mutation in the proband, chorionic villus samples were obtained during the 11th gestational week for prenatal diagnosis. Following DNA extraction, CRYAA gene mutations were also detected by PCR and DNA sequencing.

\section{Exclusion of maternal contamination}

Maternal tissue contamination was excluded using a Promega PowerPlex 16 HS System kit (Promega, Madison, WI, USA). The product was subjected to fragment analysis using an ABI3130Xl gene sequencer and the GeneMapper v3.2 software to exclude maternal contamination. The criteria for exclusion of maternal pollution were: fetus allelic fluorescence peaks derived from both parents and no fetus point 2 fluorescence detection peak representative of the mother identified. 


\section{Follow-up}

After birth, an umbilical cord blood specimen was collected for genetic diagnosis and the neonate was followed-up for 1 year.

\section{RESULTS}

\section{Ophthalmic examination}

Inspection of the pedigree (Figure 1) suggested a clear autosomal dominant pattern of inheritance. In all patients, the optical section showed a ring with white particles, on which the outer layer was porcelain white and the inner layer was cloudy. Between the opacity zone and the fetal nucleus were concentrically arranged white punctate and spike-like opacities (Figure 2).

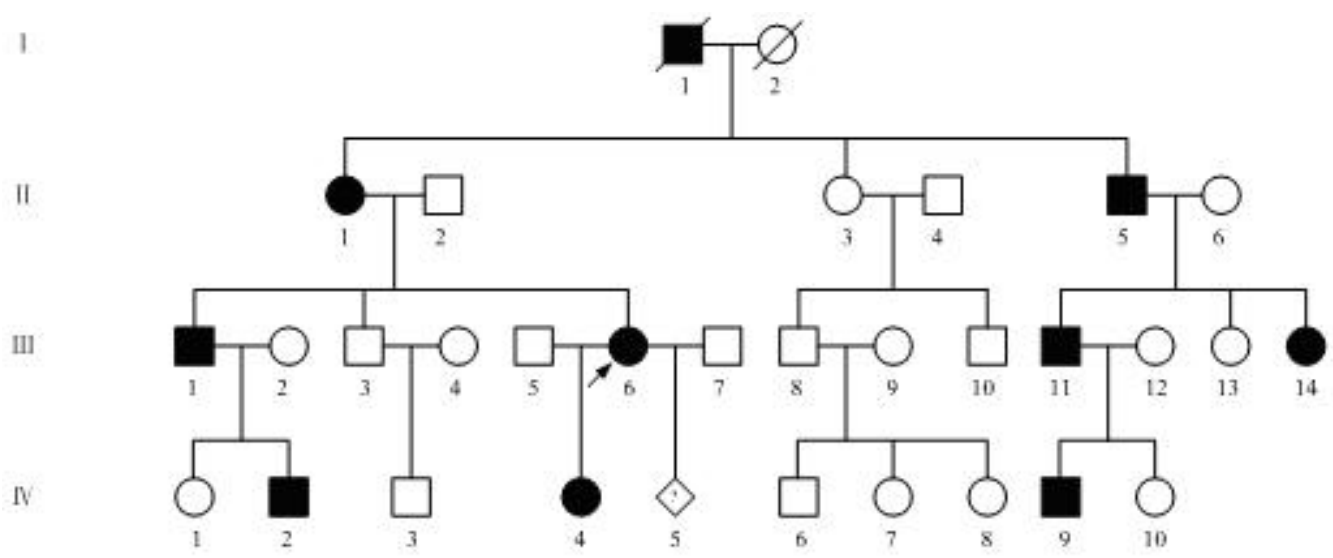

Figure 1. Pedigree of the family.
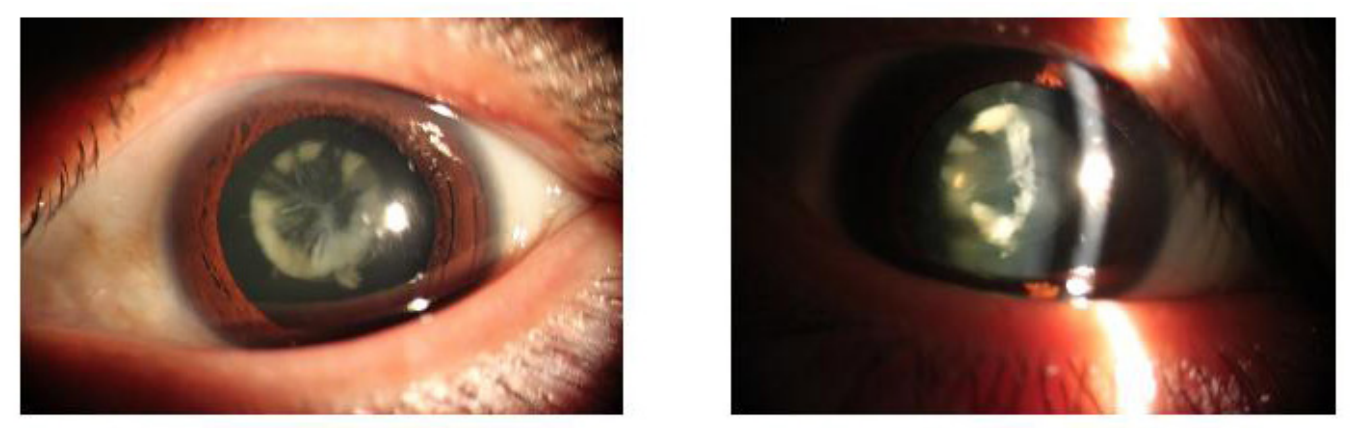

Figure 2. Slit-lamp photographs of the mother of proband (II $)$. Left: front view; Right: lateral view of the lens. 


\section{Detection of the mutation}

All patients were found to carry a heterozygous c.246_248delCGC mutation in exon 3 of the CRYAA gene, which caused removal of arginine at position 117 (p.117delR). The same mutation was not found in the 200 healthy individuals. For prenatal diagnosis, DNA sequencing suggested that the fetus did not carry the same mutation (Figure 3). The family decided to carry on with the pregnancy and a baby was born at full term. Genetic testing of umbilical cord blood taken after birth confirmed the result of the prenatal diagnosis. The neonate was followed-up for 1 year, and no cataract symptoms were observed.
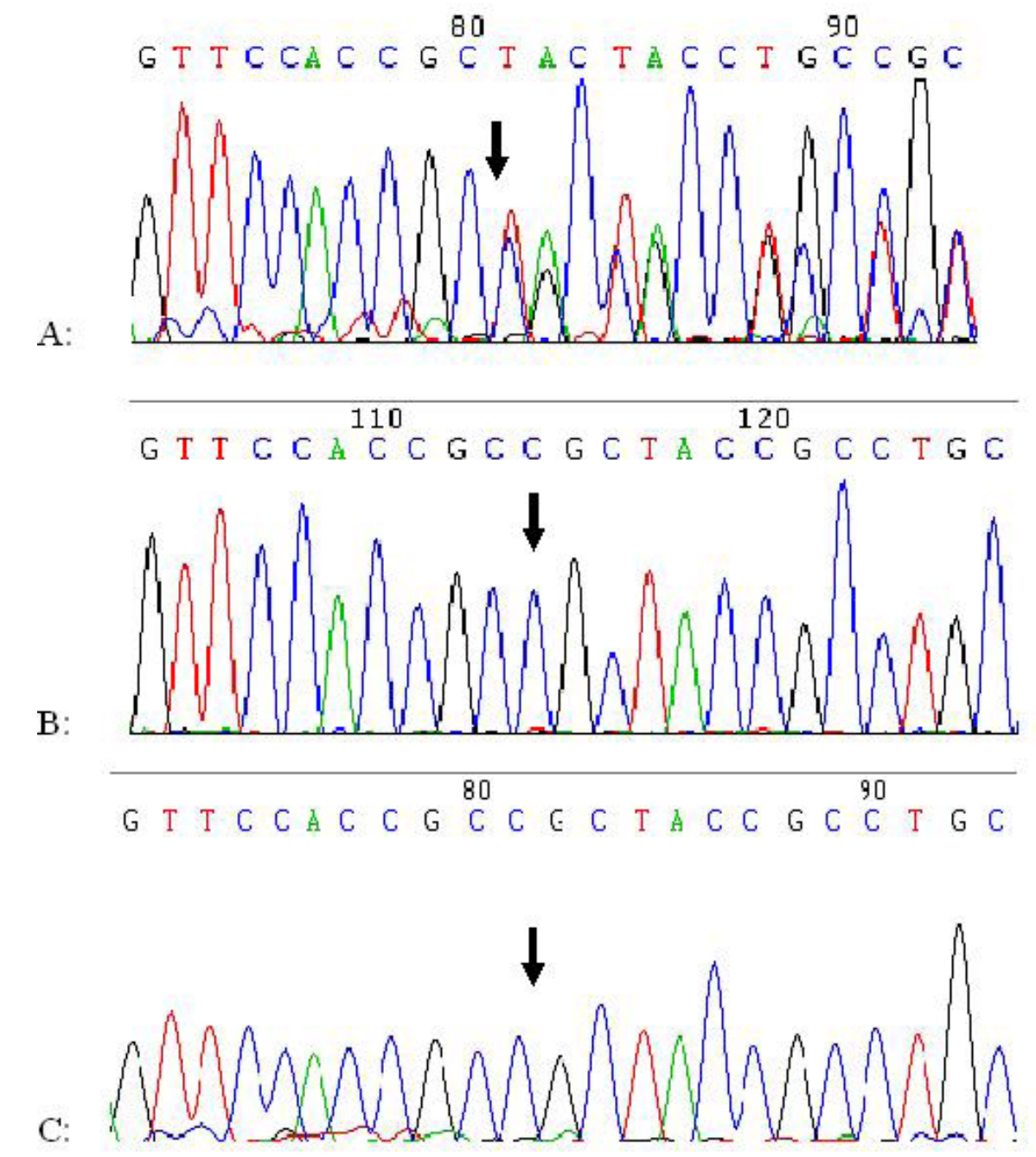

Figure 3. A. Sequencing of the CRYAA gene in the proband has identified a heterozygous c.246_248delCGC mutation, which resulted removal of arginine at position 117 (p.117delR). B. Sequence of phenotypically normal individuals. C. Sequence analysis suggested that the fetus did not carry the c.246_248delCGC mutation but a normal sequence. 


\section{Bioinformatic analysis}

As revealed by bioinformatic analysis, the removal of arginine from position 117 caused significant changes in the protein structure (Figure 4).

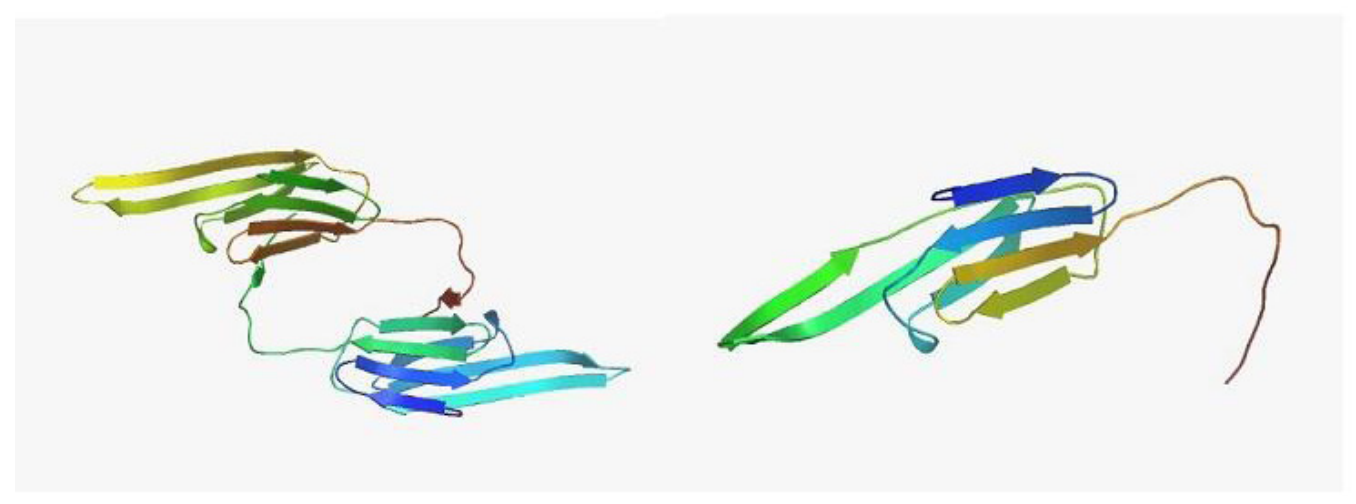

Figure 4. Structure of wide-type (left) and mutant (right) Alpha A crystallin as predicted by the SWISS-MODEL (http://beta.swissmodel.expasy.org/) (Arnold et al. 2006).

\section{Exclusion of maternal contamination}

As verified using a PowerPlex 16-HS system kit, none of the chorionic villus samples contained maternal contamination.

\section{Follow-up}

The baby was born full term. Genetic testing of the umbilical cord blood confirmed the results of the prenatal diagnosis. The neonate was followed-up for 1 year. No cataract symptoms were observed.

\section{DISCUSSION}

As the main component of the lens in vertebrates, crystallin is a major structural protein, which constitutes $90-95 \%$ of water-soluble crystalline. Regularly arranged proteins confer transparency and diopter to the lens. Human lens crystallins are divided into alpha, beta, and gamma families, among which beta and gamma crystallins are considered to be a superfamily. Alpha crystallins are composed of 2 gene products: alpha-A and alpha-B, with the letters indicating acidic and basic, respectively. Alpha crystallins can be induced by heat shock and are members of the small heat shock protein family. They act as molecular chaperones, although they do not renature proteins and release them in the fashion of a true chaperone. Instead, they hold the proteins in large soluble aggregates consisting of 30-40 subunits, with a 3:1 ratio for the alpha-A and alpha-B subunits (Graw, 2009).

The CRYAA gene has been mapped to chromosome 21q22.3 (Hawkins et al., 1987), which comprises 3 exons and encode the alpha crystalline, a protein composed of 251 amino 
acids and accounting for $9 \%$ of water-soluble crystalline. A total of 13 CRYAA mutations have been described, among which most are missense or nonsense mutations. All have been found in ADCC families except for 1 autosomal recessive case (Pras et al., 2000).

Previous studies on CRYAA gene mutations have focused on position 116. Litt et al. (1998) and Cobb and Petrash (2000) predicted that an R116C mutation (arginine to cysteine) can result in a change in polarity, along with reduced positive charge of the protein and increased mercapto groups, resulting in increased polymer hydrophobicity and protein precipitation. Experiments have confirmed that the mutation decreased the chaperone activity of the encoded protein by 4-fold. Gu et al. (2008) identified another mutation (R116H) at the same position. Similarly to R116C, this mutation caused a change in the polarity and increased hydrophobicity, but also increased its binding to lysosomes. These observations suggest that mutations in the CRYAA gene led to decreased activity as a chaperone and precipitation of the protein polymer, which is an important molecular mechanism of congenital cataract.

Notably, although our identified c.246_248delCGC mutation was an 'in-frame' mutation, which resulted in removal of a single amino acid (arginine at position 117), a significant change in the protein structure was observed. This may affect its function as a chaperone, increase the hydrophobicity of the polymer, and result in its precipitation, likely through a dominant-negative effect (or so-called "protein suicidal" phenomenon). Notably, none of our patients had microcornia, a feature that has recently been associated with congenital cataract (Liang et al., 2011). This further indicates the genotype-phenotype discrepancy of the disease.

\section{ACKNOWLEDGMENTS}

Research jointly supported by the National Natural Science Foundation of China (\#81473446), the Fundamental Research Fund for Central Universities (\#N110804002), and the Overseas Teacher Project of Ministry of Education and the State Administration of Foreign Experts Affairs (\#MS2011DBDX021).

\section{REFERENCES}

Arnold K, Bordoli L, Kopp J and Schwede T (2006). The SWISS-MODEL workspace: a web-based environment for protein structure homology modelling. Bioinformatics 22: 195-201.

Cobb BA and Petrash JM (2000). Structural and functional change in the alpha A-crystallin R116C mutant in hereditary cataracts. Biochemistry 39: 15791-15798.

Graw J (2009). Genetics of crystallins: cataract and beyond. Exp. Eye Res. 88: 173-189.

Gu F, Luo W, Li X, Wang Z, et al. (2008). A novel mutation in AlphaA-crystallin (CRYAA) caused autosomal dominant congenital cataract in a large Chinese family. Hum. Mutat. 29: 769.

Guo ZD, Hu Q, Li X, Wu Q, et al. (2011). Screen of crystallin protein mutation genes in a hereditary congenital sutural cataract family. Biomagnetism 11: 4542-4544 (in Chinese).

Hawkins JW, Van Keuren ML, Piatigorsky J, Law ML, et al. (1987). Confirmation of assignment of the human alpha-1crystallin gene (CRYA1) to chromosome 21 with regional localization to q22.3. Hum. Genet. 76: 375-380.

Hejtmancik JF and Smaoui N (2003). Molecular genetics of cataract. Dev. Ophthalmol. 37: 67-82.

Lambert SL and Drack AV (1996). Infantile cataracts. Surv. Ophthalmol. 40: 427-458.

Liang XF, Xiao W, Shi L, Hua R, et al. (2011). CRYAA gene mutation study in a family with autosomal dominant congenital cataract combined with microcornea. Zhonghua Yan Ke Za Zhi 47: 310-313 (in Chinese).

Litt M, Kramer P, LaMorticella DM, Murphey W, et al. (1998). Autosomal dominant congenital cataract associated with a missense mutation in the human alpha crystallin gene CRYAA. Hum. Mol. Genet. 7: 471-474.

Pras E, Frydman M, Levy-Nissenbaum E, Bakhan T, et al. (2000). A nonsense mutation (Wqx) in CRYAA causes autosomal recessive cataract in an inbred Jewish Dersian family. Invest. Ophthalmol. Vis. Sci. 41: 3511-3515.

Santana A and Waiswo M (2011). The genetic and molecular basis of congenital cataract. Arq. Bras. Oftalmol. 74: 136-142.

Genetics and Molecular Research 14 (1): 426-432 (2015)

CFUNPEC-RP www.funpecrp.com.br 